\title{
On a class of coupled elliptic systems in $\mathbb{R}^{N}$
}

\author{
Liliane A. MAIA and Elves A. B. SILVA \\ Departamento de Matemática, \\ Universidade de Brasília, \\ Brasília, DF 70910-900, Brazil \\ e-mails: lilimaia@unb.br, elves@unb.br
}

\begin{abstract}
It is established the existence and multiplicity of solutions for a class of coupled semilinear elliptic systems in $\mathbb{R}^{N}$ where none of the potentials are coercive. The main goal is to consider systems where the primitive of the nonlinearity is superquadratic on appropriate directions.
\end{abstract}

2000 Mathematics Subject Classification: 35J50, 35J55.

Key words: Elliptic systems, Variational methods, superquadratic nonlinearity.

\section{Introduction}

In this paper we present results on the existence and multiplicity of solutions for the system

$$
\left\{\begin{array}{l}
-\Delta u+a(x) u=F_{u}(x, u, v), \quad x \in \mathbb{R}^{N} \\
-\Delta v+b(x) v=F_{v}(x, u, v), \quad x \in \mathbb{R}^{N}
\end{array}\right.
$$

where $N \geq 3$ and the potentials $a$ and $b$ are continuous functions satisfying

$\left(A_{1}\right)$ there are constants $a_{0}, b_{0}>0$ such that $a(x) \geq a_{0}, b(x) \geq b_{0}$ for all $x \in \mathbb{R}^{N}$, $\left(A_{2}\right) \mu\left(\left\{x \in \mathbb{R}^{N}: a(x) b(x)<M\right\}\right)<\infty$, for every $M>0$.

Here $\mu$ denotes the Lebesgue measure in $\mathbb{R}^{N}$. We also suppose that $F \in C^{1}\left(\mathbb{R}^{N} \times\right.$ $\left.\mathbb{R}^{2}, \mathbb{R}\right)$ satisfies $F(x, 0,0) \equiv 0$ and the following subcritical coupling condition

The first author was supported by CAPES/Brazil and Pronex. The second author was supported by CNpq/Brazil, Pronex and FINATEC. 
$\left(F_{1}\right)$ there are constants $c_{1}, q_{1}, q_{2}>0, p_{1}, p_{2}>1$ and functions $\alpha, \beta \in L^{\infty}\left(\mathbb{R}^{N}\right)$ such that

$$
\begin{array}{ll}
\left|F_{u}(x, z)\right| \leq c_{1}|u|^{p_{1}-1}|v|^{q_{1}}+\alpha(x)|u|+c_{1}|v|, & \forall(x, z) \in \mathbb{R}^{N} \times \mathbb{R}^{2}, \\
\left|F_{v}(x, z)\right| \leq c_{1}|u|^{q_{2}}|v|^{p_{2}-1}+c_{1}|u|+\beta(x)|v|, & \forall(x, z) \in \mathbb{R}^{N} \times \mathbb{R}^{2},
\end{array}
$$

where $z=(u, v), 2 \leq p_{i}+q_{i}<2^{*} \equiv 2 N /(N-2), i=1,2$ and $\alpha$ and $\beta$ satisfy

$$
\limsup _{|x| \rightarrow \infty} \alpha(x)=\alpha_{\infty}<a_{0} \text { and } \quad \limsup _{|x| \rightarrow \infty} \beta(x)=\beta_{\infty}<b_{0} .
$$

We observe that there is an extensive bibliography on the study of variational elliptic systems (see $[3,12,14]$ and references therein). In two recent articles $[4,6]$, the problem $(\mathrm{P})$ has been studied under coupled-resonant conditions. The main objective in this work is to complement the results in $[4,6]$ by considering systems with coupling in $\mathbb{R}^{N}$ which allow, in particular, a superquadratic growth on the primitive $F(x, z)$ on directions $z=(u, v), u \neq 0, v \neq 0$. In our first two results, we assume the following version of the Ambrosetti-Rabinowitz's $[1,8]$ superquadratic condition.

$\left(F_{2}\right)$ there are constants $c_{2}>0, \theta>2, p, q, \mu, \nu \geq 0$, and functions $h_{1}, h_{2} \in$ $L^{1}\left(\mathbb{R}^{N}\right)$ such that $2 \leq \mu+\nu, p+q<2^{*}, \mu+\nu>\frac{N}{2}(p+q-2)$ (if $p+q>2$ and $\mu>p[\nu>q]$, it is supposed $\nu p>\mu(q-2)[\mu q>\nu(p-2)])$,

$$
\begin{array}{ll}
F(x, z)-\frac{1}{\theta} \nabla F(x, z) \cdot z \leq c_{2}|u|^{p}|v|^{q}+h_{1}(x), & \forall(x, z) \in \mathbb{R}^{N} \times \mathbb{R}^{2}, \\
\frac{1}{2} \nabla F(x, z) \cdot z-F(x, z) \geq c_{2}|u|^{\mu}|v|^{\nu}-h_{2}(x), & \forall(x, z) \in \mathbb{R}^{N} \times \mathbb{R}^{2} .
\end{array}
$$

The hypothesis $\left(F_{2}\right)$ is a version for our setting of a condition introduced in [12] (see also [3] for a related condition) to study a class of integro-differential problem on bounded domains of $\mathbb{R}^{N}$. We note that $c_{2}>0$ and the second relation in $\left(F_{2}\right)$ imply that $F(x, t z) / t^{2} \rightarrow \infty$, as $t \rightarrow \infty$, for every $z=(u, v) \in \mathbb{R}^{2}, u \neq 0$, $v \neq 0$ (see section 3). Considering the coupled linear problem

$$
\begin{cases}-\Delta u+a(x) u=\lambda v, & x \in \mathbb{R}^{N}, \\ -\Delta v+b(x) v=\lambda u, & x \in \mathbb{R}^{N}\end{cases}
$$

we denote by $\lambda_{1}$ the first positive eigenvalue for the problem (LP) (we refer the reader to [6] for a study of the eigenvalues of (LP)). Assuming

$\left(F_{3}\right)$ there is $\lambda \in\left[0, \lambda_{1}\right)$ such that

$$
F(x, z)-\lambda u v=o\left(|z|^{2}\right) \text {, as }|z| \rightarrow 0, \text { uniformly in } \mathbb{R}^{N},
$$

we may state: 
Theorem 1.1 Suppose $\left(A_{1}\right)$ and $\left(A_{2}\right)$ hold. If $F$ satisfies $\left(F_{1}\right)-\left(F_{3}\right)$, then problem $(P)$ possesses a nonzero solution.

In our next result we verify the existence of infinitely many solutions for $(\mathrm{P})$ under the presence of symmetry. More specifically, we suppose

$\left(F_{4}\right) F(x, z)$ is even with respect to the variable $z \in \mathbb{R}^{2}$. Under this condition, we are able to prove

Theorem 1.2 Suppose $\left(A_{1}\right)$ and $\left(A_{2}\right)$ hold. If $F$ satisfies $\left(F_{1}\right),\left(F_{2}\right)$ and $\left(F_{4}\right)$, then problem $(P)$ possesses infinitely many pairs of nonzero solutions.

The existence of a nontrivial solution for the scalar problem in $\mathbb{R}^{N}$ has been proved by Rabinowitz [9] for subcritical nonlinear terms with superlinear growth and coercive potentials. Afterwards Bartsch and Wang [2] studied the scalar problem under conditions similar to those in [2] [9] and a version of condition $\left(A_{2}\right)$ for that setting. We also observe that this hypothesis has been used in [5] to generalize to $\mathbb{R}^{N}$ the results on double resonant elliptic problems under a local nonquadraticity condition which had been proved in [7].

Theorems 1.1 and 1.2 may be seen as versions for elliptic systems with coupling of the corresponding results in $[9,2]$. We note that the condition $\left(A_{2}\right)$ allows a setting where none of potentials are coercive or satisfy the condition introduced in [2].

In our final two results we establish versions of Theorems 1.1 and 1.2 when $c_{2}$, given by $\left(F_{2}\right)$, is null. In this case the second relation in $\left(F_{2}\right)$ is not necessary:

$\left(\widehat{F_{2}}\right)$ there are $\theta>2$ and a function $h_{1} \in L^{1}\left(\mathbb{R}^{N}\right)$ such that

$$
F(x, z)-\frac{1}{\theta} \nabla F(x, z) \cdot z \leq h_{1}(x), \quad \forall(x, z) \in \mathbb{R}^{N} \times \mathbb{R}^{2} .
$$

Assuming

$\left(F_{5}\right)$ there is $c_{3} \geq 0$ such that

$$
\sup _{t \geq 0} \int_{\mathbb{R}^{N}}\left[\lambda_{1} t^{2} u_{1} v_{1}-F\left(x, t z_{1}\right)\right] d x \leq c_{3}<\infty
$$

where $z_{1}=\left(u_{1}, v_{1}\right)$ is the eigenfunction of (LP) associated with $\lambda_{1}$, we may state:

Theorem 1.3 Suppose $\left(A_{1}\right)$ and $\left(A_{2}\right)$ hold. If $F$ satisfies $\left(F_{1}\right),\left(\widehat{F_{2}}\right),\left(F_{3}\right)$ and $\left(F_{5}\right)$, then problem $(P)$ possesses a nonzero solution.

Moreover, supposing $F$ satisfies $\left(F_{4}\right)$ and

$\left(F_{6}\right)$ there exist $(a, b) \in \mathbb{R}^{2} \backslash\{(0,0)\}$ and an open subset $\Omega$ of $\mathbb{R}^{N}$ such that

$$
\frac{F(x, t a, t b)}{t^{2}} \rightarrow \infty \text {, as } t \rightarrow \infty \text {, uniformly for } x \in \Omega \text {, }
$$

we obtain: 
Theorem 1.4 Suppose $\left(A_{1}\right)$ and $\left(A_{2}\right)$ hold. If $F$ satisfies $\left(F_{1}\right),\left(\widehat{F_{2}}\right),\left(F_{4}\right)$ and $\left(F_{6}\right)$, then problem $(P)$ possesses infinitely many pairs of nonzero solutions.

\section{Preliminary results}

In order to apply the variational method to study (P), we follow Rabinowitz [9] and we set $E=E_{a} \times E_{b}$ with $E_{a}=\left\{u \in W^{1,2}\left(\mathbb{R}^{N}, \mathbb{R}\right): \int_{\mathbb{R}^{N}}\left(|\nabla u|^{2}+a(x) u^{2}\right) d x<\right.$ $\infty\}$ and $E_{b}=\left\{v \in W^{1,2}\left(\mathbb{R}^{N}, \mathbb{R}\right): \int_{\mathbb{R}^{N}}\left(|\nabla v|^{2}+b(x) v^{2}\right) d x<\infty\right\}$ endowed with the inner product

$$
\langle(u, v),(\phi, \psi)\rangle=\int_{\mathbb{R}^{N}}(\nabla u \nabla \phi+\nabla v \nabla \psi+a(x) u \phi+b(x) v \psi) d x,
$$

and associated norm denoted by $\|\cdot\|$. Standard arguments show that the associated functional $I: E \rightarrow \mathbb{R}$, defined by

$$
I(z)=\frac{1}{2}\|z\|^{2}-\int_{\mathbb{R}^{N}} F(x, z) d x, \quad \forall z=(u, v) \in E,
$$

is well defined and of class $C^{1}$. Moreover the critical points of $I$ are precisely the weak solutions of the system $(P)$. The condition $\left(A_{1}\right)$ and the Sobolev Theorem imply that the immersion $E \hookrightarrow L^{s}\left(\mathbb{R}^{N}, \mathbb{R}\right) \times L^{s}\left(\mathbb{R}^{N}, \mathbb{R}\right)$ is continuous for $2 \leq s \leq$ $2^{*}$. However, since we are not supposing that $a$ or $b$ are coercive, i.e. $a(x)$ or $b(x)$ go to $+\infty$ as $|x| \rightarrow 0$, this embedding may not be compact.

Now we will introduce some preliminary results concerning a version of the Palais-Smale condition for the functional $I$ given by (2). Let $E$ be a real Banach space and $I: E \rightarrow \mathbb{R}$ a functional of class $C^{1}$. We recall that a sequence $\left(z_{n}\right) \subset E$ is said to be a strong Cerami $[(\mathrm{SCe})]$ sequence $[13,4,6]$ if $I\left(z_{n}\right) \rightarrow c$ and $I^{\prime}\left(z_{n}\right) \rightarrow 0$ as $n \rightarrow \infty$, and $\left\|z_{n}\right\|\left\|I^{\prime}\left(z_{n}\right)\right\|$ is bounded. The functional $I$ satisfies the strong Cerami condition [(SCe)] if any strong Cerami sequence $\left(z_{n}\right) \subset E$ possesses a convergent subsequence.

The following result is proved in [6] (see the proof of Theorem 2).

Lemma 2.1 Suppose $\left(A_{1}\right)$ and $\left(A_{2}\right)$ hold. If $F$ satisfies $\left(F_{1}\right)$, then every bounded (SCe) sequence possesses a convergent subsequence.

Based on the above result, we may state

Lemma 2.2 Suppose $\left(A_{1}\right)$ and $\left(A_{2}\right)$ hold. If $F$ satisfies $\left(F_{1}\right)$ and $\left(F_{2}\right)$ or $\left(\widehat{F_{2}}\right)$, then the functional I satisifes ( $S C e$ ).

Proof. Let $\left(z_{n}\right)=\left(\left(u_{n}, v_{n}\right)\right) \subset E$ be a (SCe) sequence, i.e., $\left(z_{n}\right)$ satisfies (i) $I\left(z_{n}\right) \rightarrow c$ and $I^{\prime}\left(z_{n}\right) \rightarrow 0$ as $n \rightarrow \infty$, and (ii) $\left\|z_{n}\right\|\left\|I^{\prime}\left(z_{n}\right)\right\| \leq M<\infty$. By 
Lemma 2.1, it suffices to show that $\left(z_{n}\right)$ is a bounded sequence. Supposing $F$ satisfies $\left(F_{2}\right)$, we apply the relations (i) and (ii) to find $M_{1}>0$ such that

$$
\begin{aligned}
c_{2} \int_{\mathbb{R}^{N}}\left|u_{n}\right|^{\mu}\left|v_{n}\right|^{\nu} d x \leq I\left(z_{n}\right)-\frac{1}{2}\left\langle I^{\prime}\left(z_{n}\right), z_{n}\right\rangle+\left\|h_{2}\right\|_{L^{1}} \leq M_{1}, \\
\left(\frac{1}{2}-\frac{1}{\theta}\right)\left\|z_{n}\right\|^{2} \leq I\left(z_{n}\right)-\frac{1}{\theta}\left\langle I^{\prime}\left(z_{n}\right), z_{n}\right\rangle+c_{2} \int_{\mathbb{R}^{N}}\left|u_{n}\right|^{p}\left|v_{n}\right|^{q} d x+\left\|h_{1}\right\|_{L^{1}} \\
\leq c_{2} \int_{\mathbb{R}^{N}}\left|u_{n}\right|^{p}\left|v_{n}\right|^{q} d x+M_{1} .
\end{aligned}
$$

Considering $\gamma \equiv p+q$ and $\eta \equiv \mu+\nu$, we apply $\left(F_{2}\right)$ to find $r>1$ such that $r p \geq \mu, r q \geq \nu, 2(r-1) \leq \gamma r-\eta \leq 2^{*}(r-1)$ and $(\gamma r-\eta) / r<2$. Since $\left|u_{n}(x)\right|,\left|v_{n}(x)\right| \leq\left|z_{n}(x)\right|$, for every $x \in \mathbb{R}^{N}$, we may write

$$
\int_{\mathbb{R}^{N}}\left|u_{n}\right|^{p}\left|v_{n}\right|^{q} d x \leq \int_{\mathbb{R}^{N}}\left(\left|u_{n}\right|^{\mu}\left|v_{n}\right|^{\nu}\right)^{\frac{1}{r}}\left|z_{n}\right|^{\frac{\gamma r-\eta}{r}} d x .
$$

Applying Holder's inequality to the above relation, we get

$$
\int_{\mathbb{R}^{N}}\left|u_{n}\right|^{p}\left|v_{n}\right|^{q} d x \leq\left[\int_{\mathbb{R}^{N}}\left|u_{n}\right|^{\mu}\left|v_{n}\right|^{\nu} d x\right]^{\frac{1}{r}}\left[\int_{\mathbb{R}^{N}}\left|z_{n}\right|^{\frac{\gamma r-\eta}{r-1}} d x\right]^{\frac{r-1}{r}} .
$$

Hence, by the inequality (3), there is $M_{2}>0$ such that

$$
\int_{\mathbb{R}^{N}}\left|u_{n}\right|^{p}\left|v_{n}\right|^{q} d x \leq M_{2}\left\|z_{n}\right\|_{L^{\frac{\gamma r-\eta}{r-1}}}^{\frac{\gamma r-\eta}{r}} .
$$

Since $\frac{\gamma r-\eta}{r-1} \in\left[2,2^{*}\right]$, from the embedding $E \hookrightarrow L^{s}\left(\mathbb{R}^{N}, \mathbb{R}\right) \times L^{s}\left(\mathbb{R}^{N}, \mathbb{R}\right), 2 \leq s \leq 2^{*}$, and $\theta>2$, by the above inequality and (4), we find $M_{3}, M_{4}>0$ such that

$$
\left\|z_{n}\right\|^{2} \leq M_{3}\left\|z_{n}\right\|^{\frac{\gamma r-\eta}{r}}+M_{4}
$$

Noting that by our choice of $r$ we have $\frac{\gamma r-\eta}{r}<2$, we may conclude that $\left(z_{n}\right)$ is a bounded sequence. When $\left(\widehat{F_{2}}\right)$ holds, the conclusion that $\left(z_{n}\right)$ is bounded is a direct consequence of (4) with $c_{2}=0$. This concludes the proof of the lemma.

Next, we state the versions of the abstract results in [1] which will be used in our proofs of Theorems 1.1-1.2.

Theorem $2.3([\mathbf{1 0}, \mathbf{1 1}])$ Let $E$ be a real Banach space. Suppose $I \in C^{1}(E, \mathbb{R})$ satisfies $I(0)=0,(S C e)$ and

$\left(I_{1}\right)$ there exists $\rho>0$ such that $I(z) \geq 0$, for all $z \in \partial B_{\rho}(0)$.

$\left(I_{2}\right)$ there exist $\beta \in \mathbb{R}$ and $e \in E$ such that $I($ te $) \leq \beta$ for every $t \geq 0$.

Then I possesses a nonzero critical point. 
Theorem 2.4 ([10, 15]) Let $E=V \oplus W$ be a real Hilbert space with $V$ finite dimensional and $W=V^{\perp}$. Suppose $I \in C^{1}(E, \mathbb{R})$ is even and satisfies $I(0)=0$, (SCe) and

$\left(I_{3}\right)$ there exists $\rho>0$ such that $I(z) \geq 0$, for all $z \in B_{\rho}(0) \cap W$.

$\left(I_{4}\right)$ there exists a finite dimensional closed subspace $\widehat{V}$ of $E$ and $\beta \in \mathbb{R}$ such that $\widehat{V} \supset V$ and $I(z) \leq \beta$, for all $z \in \widehat{V}$,

Then I possesses $\operatorname{dim} \widehat{V}-\operatorname{dim} V$ pairs of nontrivial critical points.

Remark 2.5 Theorems 2.3 and 2.4 in $[10,11]$ and $[10,15]$ are proved for the Palais-Smale condition $[1,8]$. The version for the (SCe) condition is based on a deformation lemma proved in [13].

\section{Proofs of theorems 1.1 and 1.3}

The proof of Theorem 1.1 will be accomplished by verifying that the functional $I$, given by (2), satisfies the hypotheses of Theorem 2.3 . We start by recalling the variational characterization of the eigenvalue $\lambda_{1}$ :

$$
\lambda_{1}=\sup \left\{\mu \geq 0:\|z\|^{2}-2 \mu \int_{\mathbb{R}^{N}} u v d x \geq 0, \forall z \in E\right\} .
$$

Consequently, taking $\lambda \in\left[0, \lambda_{1}\right)$, given by $\left(F_{3}\right)$, we find $c_{3}>0$ such that

$$
\frac{1}{2}\|z\|^{2}-\lambda \int_{\mathbb{R}^{N}} u v d x \geq c_{3}\|z\|^{2}, \forall z \in E .
$$

Now, given $\epsilon>0$, we use $\left(F_{3}\right)$ to find $\delta>0$ such that

$$
F(x, z)-\lambda u v \leq \epsilon|z|^{2}, \forall(x, z) \in \mathbb{R}^{N} \times \mathbb{R}^{2},|z| \leq \delta .
$$

Furthermore, observing that $F(x, 0,0) \equiv 0$ and applying $\left(F_{1}\right)$, we obtain constants $c_{4}>0$ and $2<\sigma<2^{*}$ such that

$$
F(x, z)-\lambda u v \leq c_{4}|z|^{\sigma}, \forall(x, z) \in \mathbb{R}^{N} \times \mathbb{R}^{2},|z|>\delta .
$$

Combining these two estimates, we get

$$
F(x, z)-\lambda u v \leq \epsilon|z|^{2}+c_{4}|z|^{\sigma}, \forall(x, z) \in \mathbb{R}^{N} \times \mathbb{R}^{2} .
$$

Consequently, by definition (2) and inequality (6),

$$
I(z) \geq c_{3}\|z\|^{2}-\epsilon\|z\|_{L^{2}}^{2}-c_{4}\|z\|_{L^{\sigma}}^{\sigma}, \forall z \in E .
$$


Using the embedding $E \hookrightarrow L^{s}\left(\mathbb{R}^{N}, \mathbb{R}\right) \times L^{s}\left(\mathbb{R}^{N}, \mathbb{R}\right), 2 \leq s \leq 2^{*}, \sigma>2$ and choosing $\epsilon>0$ sufficiently small, we conclude that $I$ satisfies the condition $\left(I_{1}\right)$.

Now we shall verify the condition $\left(I_{2}\right)$ when $\eta=\mu+\nu>2$ (the case $\eta=2$ is proved by a similar argument). Given $(x, z) \in \mathbb{R}^{N} \times \mathbb{R}^{2}$, we consider the function $g(t)=F(x, t z)$, for $t \geq 0$, and apply the second relation in $\left(F_{2}\right)$ to get

$$
F(x, t z) \geq t^{2} F(x, z)+\frac{2 c_{2}|u|^{\mu}|v|^{\nu}}{\eta-2}\left(t^{\eta}-t^{2}\right)-\left|h_{2}(x)\right|\left(t^{2}-1\right), \quad \forall t \geq 1 .
$$

Now we fix $\varphi \in C_{0}^{\infty}\left(\mathbb{R}^{N}\right), \varphi \not \equiv 0$, and take $e=(\varphi, \varphi)$. Using the above relation, we get

$$
\begin{gathered}
\int_{\mathbb{R}^{N}} F(x, t e) d x \geq t^{2} \int_{\mathbb{R}^{N}} F(x, e) d x+\frac{2 c_{2}\|\varphi\|_{L^{\eta}}^{\eta}}{\eta-2}\left(t^{\eta}-t^{2}\right)-\left(t^{2}-1\right)\left\|h_{2}\right\|_{L^{1}}, \\
\forall t \geq 1 .
\end{gathered}
$$

Since $\eta>2$, we conclude that

$$
\frac{1}{t^{2}} \int_{\mathbb{R}^{N}} F(x, t e) d x \rightarrow \infty, \text { as } t \rightarrow \infty
$$

The definition (2) and the limit in (7) show that the functional $I$ satisfies $\left(I_{2}\right)$ whenever the condition $\left(F_{2}\right)$ holds. Since $I$ satisfies $I(0)=0,\left(I_{1}\right),\left(I_{2}\right)$ and (SCe), by Lemma 2.2, we may apply the Theorem 2.3 to conclude that problem (P) possesses a nonzero solution. This concludes the proof of Theorem 1.1.

For proving the Theorem 1.3, we just need to verify that the functional $I$ satisfies the condition $\left(I_{2}\right)$. But, taking $e=z_{1}$ and noting that $\left\|z_{1}\right\|^{2}=$ $2 \lambda_{1} \int_{\mathbb{R}^{N}} u_{1} v_{1} d x$, we obtain that $\left(I_{2}\right)$ follows directly from $\left(\hat{F}_{z}\right)$.

\section{Proofs of theorems 1.2 and 1.4}

We will prove Theorem 1.2 by verifying that the functional $I$ satisfies the hypotheses of Theorem 2.4 with $\operatorname{dim} \widehat{V}-\operatorname{dim} V$ as large as we like. Let $\left\{z_{1}, z_{2}, \cdots, z_{k}, \cdots\right\}$ be an orthonormal basis for $E$. We set $E_{k}=\operatorname{span}\left\{z_{1}, z_{2}, \cdots, z_{k}\right\}$ for every $k \in \mathbb{N}$. In order to prove Theorem 1.2 we need the following technical result.

Lemma 4.6 Suppose $\left(A_{1}\right)$ and $\left(A_{2}\right)$ hold. If $p, q>0$ and $p+q \geq 2$; then, given $\delta>0$, there is $k_{0} \in \mathbb{N}$ such that

$$
\int_{\mathbb{R}^{N}}|u|^{p}|v|^{q} d x \leq \delta\|z\|^{p+q}, \forall z \in E_{k}^{\perp}, k \geq k_{0} .
$$


Proof. First, we suppose that $p=q=1$. Arguing by contradiction, we suppose there exist $c>0$, and a sequence $z_{k}=\left(u_{k}, v_{k}\right) \subset E_{k}^{\perp}$ such that, for every $k \in \mathbb{N}$, $\left\|z_{k}\right\|=1$ and

$$
\int_{\mathbb{R}^{N}}\left|u_{k}\right|\left|v_{k}\right| d x \geq c>0
$$

Without loss of generality, we may suppose that

$$
\left\{\begin{array}{l}
z_{k} \rightarrow 0, \text { in } E \\
u_{k}, v_{k} \rightarrow 0, \text { in } L_{l o c}^{s}\left(\mathbb{R}^{N}\right) \text { for } 2 \leq s<2^{*} .
\end{array}\right.
$$

By the condition $\left(A_{2}\right)$, given $\varepsilon>0$ we may choose $R>0$ such that $\mu\left(C_{\varepsilon}\right)<\varepsilon$, where $C_{\varepsilon}=\left\{x \in \mathbb{R}^{N} \backslash B_{R}(0): a(x) b(x) \leq \varepsilon^{-2}\right\}$. Invoking (9) and Holder's inequality, we obtain $M>0$ such that

$$
\int_{C_{\epsilon}}\left|u_{k}\left\|v_{k} \mid d x \leq \mu\left(C_{\epsilon}\right)^{\frac{2}{N}}\right\| u_{k}\left\|_{L^{2^{*}}}\right\| v_{k} \|_{L^{2^{*}}} \leq M \mu\left(C_{\epsilon}\right)^{\frac{2}{N}} \leq M \epsilon^{\frac{2}{N}} .\right.
$$

Now, setting $D_{\varepsilon}=\mathbb{R}^{N} \backslash\left(B_{R}(0) \cup C_{\varepsilon}\right)$, we may use (9) and Holder's inequality one more time to get

$$
\int_{D_{\varepsilon}}\left|u_{k}\right|\left|v_{k}\right| d x \leq \varepsilon \int_{D_{\varepsilon}} \sqrt{a(x)}\left|u_{k}\right| \sqrt{b(x)}\left|v_{k}\right| d x \leq \varepsilon .
$$

Moreover, by (9), for $k$ sufficiently large,

$$
\int_{B_{R}(0)}\left|u_{k}\right|\left|v_{k}\right| d x<\varepsilon
$$

In view of (10)-(12) and the fact that $\varepsilon>0$ can be chosen arbitrarily small, we obtain a contradiction to (8). This proves the lemma for $p=q=1$. Now we consider the general case. Supposing $p \geq q$, we claim that there is $c_{5}>0$ such that

$$
\int_{\mathbb{R}^{N}}|u|^{p}|v|^{q} d x \leq c_{5}\|u v\|_{L^{\frac{p+q}{2}}}^{q}\|z\|^{p-q}, \forall z \in E .
$$

The above inequality is trivially true for $p=q$. Hence, we may assume that $p>q$. Applying Holder's inequality, we get

$$
\int_{\mathbb{R}^{N}}|u|^{p}|v|^{q} d x=\int_{\mathbb{R}^{N}}(\sqrt{|u||v|})^{2 q}|u|^{p-q} d x \leq\|u v\|_{L^{\frac{p+q}{2}}}^{q}\|u\|_{L^{p+q}}^{p-q} .
$$

Using that $|u(x)| \leq|z(x)|$, for every $x \in \mathbb{R}^{N}$, and the appropriate embedding, we obtain (13). This proves the claim. Taking $t \in(0,1]$ such that $p+q=2 t+(1-t) 2^{*}$, 
by interpolation, we obtain

$$
\begin{aligned}
\int_{\mathbb{R}^{N}}(|u||v|)^{\frac{p+q}{2}} d x & \leq\left(\int_{\mathbb{R}^{N}}|u v| d x\right)^{t}\left(\int_{\mathbb{R}^{N}}|u v|^{2^{*} / 2} d x\right)^{1-t} \\
& \leq\left(\int_{\mathbb{R}^{N}}|u v| d x\right)^{t}\|z\|_{L^{2^{*}}}^{2^{*}(1-t)}
\end{aligned}
$$

Finally, given $\delta>0$, using the above inequality and the fact that the lemma holds for $p=q=1$, we obtain $k_{0} \in \mathbb{N}$ such that

$$
\|u v\|_{L^{\frac{p+q}{2}}}^{q} \leq\left(c_{5}\right)^{-1} \delta\|z\|^{2 q}, \forall z \in E_{k}^{\perp}, k \geq k_{0}
$$

Combining the above inequality and (13), we conclude the proof of the lemma.

The next result follows from condition (1) and the fact that the embedding $E \hookrightarrow L^{s}(U) \times L^{s}(U), 2 \leq s<2^{*}$, is compact on bounded open subsets $U \subset \mathbb{R}^{N}$.

Lemma 4.7 Suppose $\left(A_{1}\right)$ and $\left(A_{2}\right)$ hold. If $\alpha$ and $\beta \in L^{\infty}\left(\mathbb{R}^{N}\right)$ satisfy $(1)$, then there is $r>1$ such that, given $\delta>0$, we may find $k_{1} \in \mathbb{N}$ such that

$$
\begin{gathered}
\int_{\mathbb{R}^{N}} \alpha(x) u^{2} d x \leq \frac{1}{r} \int_{\mathbb{R}^{N}} a(x) u^{2} d x+\delta\|z\|^{2}, \forall z \in E_{k}^{\perp}, k \geq k_{1}, \\
\int_{\mathbb{R}^{N}} \beta(x) u^{2} d x \leq \frac{1}{r} \int_{\mathbb{R}^{N}} b(x) u^{2} d x+\delta\|z\|^{2}, \forall z \in E_{k}^{\perp}, k \geq k_{1} .
\end{gathered}
$$

Now we may check condition $\left(I_{3}\right)$. Let $r>1$ be the constant given by Lemma 4.7. Then, given $\delta>0$, we may apply $\left(F_{1}\right)$ and Lemmas $4.6-4.7$ to get $c_{6}>0,2<\sigma<2^{*}$ and $k \in \mathbb{N}$ such that

$$
\int_{\mathbb{R}^{N}}|F(x, z)| d x \leq \frac{1}{2 r}\|z\|^{2}+\delta\|z\|^{\sigma}+c_{6}\|z\|, \forall z \in E_{k}^{\perp} .
$$

Consequently,

$$
I(z) \geq\left(\frac{r-1}{2 r}-\delta\|z\|^{(\sigma-2)}\right)\|z\|^{2}-c_{6}\|z\|, \forall z \in E_{k}^{\perp} .
$$

Taking $\rho=\rho(\delta)>0$ such that $\rho^{(\sigma-2)}=(r-1) / 4 \delta r$, we have $I(z) \geq(r-$ 1) $/(4 r) \rho^{2}-c_{6} \rho$, for every $z \in E_{k}^{\perp} \cap \partial B_{\rho}(0)$. Noting that $\rho \rightarrow \infty$ as $\delta \rightarrow 0$, by the above inequality, we may conclude that there is $k \in \mathbb{N}$ such that $\left(I_{3}\right)$ holds for $V=E_{k}$ and $W=E_{k}^{\perp}$.

Now, given $m \in \mathbb{N}$, we take $\left\{\varphi_{1}, \cdots, \varphi_{m+k}\right\} \subset C_{0}^{\infty}\left(\mathbb{R}^{N}\right)$ such that $\operatorname{supp}\left(\varphi_{i}\right) \cap$ $\operatorname{supp}\left(\varphi_{j}\right)=\emptyset$ for every $i \neq j \in\{1, \cdots m+k\}$. Invoking (7) and considering $\widehat{V}=\operatorname{span}\left\{\left(\varphi_{1}, \varphi_{1}\right) \cdots,\left(\varphi_{m+k}, \varphi_{m+k}\right)\right\}$, we see easily that the functional $I$ satisfies 
$\left(I_{4}\right)$. Since $I$ satisfies $I(0)=0,\left(I_{3}\right),\left(I_{4}\right),(\mathrm{SCe})$ and it is even, by $\left(F_{4}\right)$, we may apply Theorem 2.4 to conclude that (P) possesses $\operatorname{dim} \widehat{V}-\operatorname{dim} V=m$ pairs of nonzero solutions for every $m \in \mathbb{N}$. This concludes the proof of Theorem 1.2.

For the proof of Theorem 1.4 we need only to check condition $\left(I_{4}\right)$. We consider $\Omega$ given by $\left(F_{6}\right)$ and take $\left\{\left\{\varphi_{1}, \cdots, \varphi_{m+k}\right\}\right.$ as above with $\operatorname{supp}\left(\varphi_{i}\right) \subset \Omega$, for $1 \leq i \leq k+m$. Then, setting

$\widehat{V}=\operatorname{span}\left\{\left(a \varphi_{1}, b \varphi_{1}\right) \cdots,\left(a \varphi_{m+k}, b \varphi_{m+k}\right)\right\}$, we may apply condition $\left(F_{6}\right)$ to check that $\left(I_{4}\right)$ holds.

\section{References}

[1] A. AMBROSETTI and P. H. RABINOWITZ, Dual variational methods in critical point theory and applications, J. Funct. Anal. 14 (1973), 349-381.

[2] T. BARTSCH and Z. WANG, Existence and multiplicity results for some superlinear elliptic problems on $\mathbb{R}^{N}$, Comm. in PDE 20 (1995), 1725-1741.

[3] D. G. COSTA and C. A. MAGALHÃES, A unified approach to a class of strongly indefinite functionals, J. Diff. Equ. 125 (1996), 521-547.

[4] M. F. FURTADO, L. A. MAIA and E. A. B. SILVA, Existence and multiplicity results for some superlinear elliptic problems on $\mathbb{R}^{N}$, Comm. in PDE 27 (2002), 515-1536.

[5] M. F. FURTADO, L. A. MAIA and E. A. B. SILVA, On a double resonant problem in $\mathbb{R}^{N}$, Int. and Diff. Equ. 15 (2002), 1335-1344.

[6] M. F. FURTADO, L. A. MAIA and E. A. B. SILVA, Systems with coupling in $\mathbb{R}^{N}$ for a class of noncoercive potentials. Proc. of the Fourth International Conference on Dynamical Systems and Differential Equations, (Wilmington, 2002), Disc. and Cont. Dyn. Syst., suppl. vol. (2003), 295-304.

[7] M. F. FURTADO and E. A. B. SILVA, Double resonant problems which are locally nonquadratic at infinity, Electron. J. Diff. Equ. Conf. 06 (2001), $155-171$.

[8] P. H. RABINOWITZ, Minimax methods in critical point theory with applicationstto differential equations, CBMS Regional Conf. Ser. in Math. 65, AMS, Providence, RI, 1986.

[9] P. H. RABINOWITZ, On a class of nonlinear Schrödinger Equations, Z. Angew. Math. Phys. 42 (1992), 270-291.

[10] E. A. B. SILVA, Critical point theorems and applications to differential equations, Ph.D. thesis, University of Wisconsin-Madison, 1988. 
[11] E. A. B. SILVA, Linking theorems and applications to semilinear problems at resonance, Nonlinear Analysis 16 (1991), 455-477.

[12] E. A. B. SILVA, Existence and multiplicity of solutions for semilinear elliptic systems, Nonlinear Diff. Eq. and Appl. 1 (1994), 339-363.

[13] E. A. B. SILVA, Subharmonic solutions for subquadratic Hamiltonian systems, J. Diff. Equ. 115 (1995), 120-145.

[14] E. A. B. SILVA, Nontrivial solutions for noncooperative elliptic systems at resonance, Electron. J. Diff. Equ. Conf. 06 (2001), 267-283.

[15] E. A. B. SILVA and M. S. XAVIER, Multiplicity of solutions for quasilinear ellptic systems with critical growth, to appear in NoDEA.

Received 21 October 2005; accepted 20 September 2006

Published Online First 21 September 2007

To access this journal online: http://www.birkhauser.ch 